\title{
Psychosocial adaptation in adolescents and young adults with Marfan syndrome: an exploratory study
}

\author{
A Van Tongerloo, A De Paepe
}

\begin{abstract}
We conducted a pilot study to evaluate the psychological effects and consequences of Marfan syndrome in 17 patients between 16 and 35 years of age. Through a semi-structured interview, we investigated how the patients coped daily with Marfan syndrome and evaluated the impact of the disease on specific items such as schooling, occupational choices, selfimage, and social behaviour. A second part of the study consisted of a battery of standardised psychological tests to evaluate the patients' anxiety and depression levels and coping styles. The following psychological tests were used: State and Trait Anxiety Inventory (STAI), Beck Depression Inventory (BDI), and the Utrecht Coping List (UCL). The interviews showed that the disease represented a significant burden on the daily physical activities of the patients, as well as on their schooling and job opportunities. During childhood, most of them were insensitively teased by peers because of their typical phenotypic features. This had its consequences on their social behaviour in that they are all more introvert. In the female patients, the risk associated with child bearing represented a major concern. Most patients emphasised the need for accurate information about the illness immediately after knowing the diagnosis and all found psychological support helpful. Depression and anxiety levels were higher in the female than in the male group, without being significantly different from the normal population. Nevertheless, most patients have come to terms with their disease and consider themselves happy most of the time.

(F Med Genet 1998;35:405-409)
\end{abstract}

Keywords: Marfan syndrome; adolescents; psychological consequences; psychosocial adaptation

Department of

Medical Genetics,

University Hospital

Gent, De Pintelaan

185, 9000 Gent,

Belgium

A Van Tongerloo

A De Paepe

Correspondence to:

Dr Van Tongerloo.

Received 29 August 1997 Revised version accepted for publication 7 November 1997
Marfan syndrome is relatively common: its prevalence is estimated at 1 per 5000 . Life drome is generally reduced because of the cardiovascular complications. Good clinical
Marfan syndrome is an autosomal dominant connective tissue disorder with pleiotrop ocular, and cardiovascular systems. monary system, skin and integument, and the central nervous system may also be involved. ${ }^{1}$ management, including restriction of physical activity, reduction in emotional stress, pharmacological prevention of haemodynamic stress ( $\beta$ adrenergic blockade), and current cardiovascular repair techniques all contribute to improving the long term function and life expectancy of patients. ${ }^{2}$

Although many reports exist on the clinical manifestations, the molecular background, and the medical management of Marfan syndrome, ${ }^{3-7}$ little is known about the psychosocial impact of the disorder on the patients' quality of life. Therefore, we conducted an exploratory study with adolescents and young adults with Marfan syndrome. The overall aim of this study was to assess their psychosocial functioning, including coping styles, compliance with medical treatment and physical restrictions, self-esteem, and social contacts.

\section{Patients and methods}

PATIENTS

Between December 1995 and May 1997, 21 patients between 16 and 35 years of age were asked to participate in the study. All patients fulfilled the diagnosis of Marfan syndrome according to the revised diagnostic criteria. ${ }^{1}$ Two adult patients refused to participate because of denial of diagnosis and one parent refused for her two affected teenagers. This reduced the number to a total of 17 participants from 14 families. Ten patients were male and seven female. The mean age of the patient group was 22.6 years (SD 4.8) and ranged from 17 to 34 . Twelve patients had inherited Marfan syndrome from a parent and five were sporadic cases. Nine had a brother/sister with Marfan syndrome. One patient's brother was not examined, so the diagnosis could never be formally excluded. Other sociodemographic findings of the patient group are listed in table 1.

Sixteen of the $17(94 \%)$ patients underwent one or more illness related surgical interventions. The indications for operation are listed in table 2. One of the three patients who had received aortic/valve surgery in the past needed a cardiac transplant. At the time of the study, 10 of the $17(59 \%)$ patients took medication. Four of them were treated with a $\beta$ adrenergic blocking drug because of aortic dilatation. Other frequently used medications were eye drops, antiarrhythmics, analgesics, anxiolytics, and hypnotics.

\section{METHODS}

The first part of the study consisted of a semistructured interview in which patients were questioned about the time of diagnosis and 
Table 1 Sociodemographic findings of the patient group $(n=17)$

\begin{tabular}{ll}
\hline Education (highest level) & $\mathrm{n}=17$ \\
Secondary school ongoing & 6 \\
Secondary school & 7 \\
Higher education & 3 \\
University & 1 \\
Marital status & $\mathrm{n}=17$ \\
Living with parents, has no partner & 8 \\
Living with parents, has partner & 3 \\
Married, no children & 4 \\
Cohabiting, no children & 1 \\
Living alone, has partner & 1 \\
Type of employment & $\mathrm{n}=11$ \\
Employed & 6 \\
Unemployed & 1 \\
Disability pension & 4 \\
\hline
\end{tabular}

areas of psychosocial adjustment, including coping with the illness, compliance with medical treatment and physical restrictions, influence of the illness on childhood, educational, and career choices, and social contacts.

Furthermore, we explored their self-image and their need for professional support. We did not believe a control group to be applicable to this part of the study.

In the second part of the study, patients were offered a battery of standardised psychological tests.

Beck Depressie Vragenlijst is the Dutch adaptation of the Beck Depression Inventory (BDI). This is a 21 item instrument designed to assess the level of depression in adolescents and adults. Patients must rate each item on a four point scale, ranging from 0 to 3 in terms of severity. The maximum score is 63 . Normative data are available for comparison. ${ }^{89}$

Zelf Beoordelings Vragenlijst is the Dutch adaptation of the Spielberger State-Trait Anxiety Inventory (STAI). This instrument is a self-reporting questionnaire measuring two anxiety concepts, state anxiety (DY-1) and trait anxiety (DY-2). The concept of "state anxiety" refers to a reactive condition of situational anxiety (here, the confrontation with the disease), while "trait anxiety" stands for the more stable level of anxiety as a personality trait. Two times 20 Likert-type questions must be answered on a 1 to 4 scale with, for each concept, a maximum score of 80 . Scores are transformed into deciles. Normative data are available for comparison. ${ }^{1011}$

Utrechtse Coping Lijst (UCL) is an instrument to assess ways of appraisal and coping with daily problems and stressful life events. The instrument consists of 47 items, each of which is rated on a 1 to 4 scale. Every item refers to one of seven scales, which indicate coping behaviour: active coping, palliative coping,

Table 2 Overview of surgical interventions related to Marfan syndrome

\begin{tabular}{lll}
\hline & & Total \\
\hline Cardiovascular & & 6 \\
Aortic root + valve replacement & 2 & \\
Mitral valve repair & 2 & \\
Mitral valve replacement & 1 & \\
$\quad$ Heart transplantation & 1 & 8 \\
Ocular & 1 & \\
$\quad$ Lens extraction, unilateral & 7 & \\
$\quad$ Lens extraction, bilateral & & 12 \\
Orthopaedic & & 26 \\
Total & \\
\hline
\end{tabular}

avoidance, social support seeking, depressive reaction, expression of emotions, reassuring thoughts. Scores are transformed into centiles. Normative data are available for comparison..$^{12} 13$

Our attempt to study unaffected friends of the Marfan patients as sex and age matched control subjects was hampered by the small response rate and the fact that most patients $(n=10)$ felt uneasy about asking a friend to participate. Therefore, we compared the results of the questionnaires measuring anxiety and depression levels and coping styles with the normative scores.

\section{PROCEDURE}

All patients were interviewed by the principal investigator (AVT), who is a clinical psychologist. Fourteen patients came to the Department of Medical Genetics at the University Hospital in Gent and three (from one family) were seen at the Genetics Department at the University Hospital in Antwerp. After the interview, which took between 40 and 75 minutes, the patients were subjected to the set of psychological tests. One questionnaire (STAI) was completed immediately; the BDI and the UCL were taken home, completed there, and returned to us by mail. Another envelope containing all three questionnaires was also given to the patient with the request to ask a close friend to complete them and return them by mail in a stamped envelope.

Student's $t$ test was used to analyse the difference of means of the Marfan group and the normal control group for the depression and anxiety level and coping styles. A p value of 0.05 or less was considered significant.

\section{Results}

PART I: INTERVIEW

The diagnosis

The mean age of the subjects at diagnosis was 12.2 years (SD 7.0), range 5 to 29 years. Two brothers were diagnosed at 14 and 16 years of age respectively, after the diagnosis had been established in their younger sister of 7 years. One woman consulted for the first time after she saw a television programme on Marfan syndrome. A man was diagnosed at the age of 29 after he was admitted to hospital for cardiac failure. Eight out of $17(47 \%)$ patients were initially informed about Marfan syndrome by their parents because of a positive family history. The remaining nine $(53 \%)$ patients were given the diagnosis by a medical geneticist. These patients were usually referred to the medical geneticist by the treating physician. Information given by parents was often incomplete. Additional information, such as on the phenotypic variability of the disease, the genetic risk, and the causal defect was given by the medical geneticist.

\section{Coping with the illness}

Five $(29 \%)$ patients had never experienced any difficulties in accepting their illness and they hardly ever thought about the disease. One (6\%) 17 year old male adolescent had great coping difficulties: he had problems with 
Table 3 Reasons for applying psychological support at the Genetics Department $(n=17)$

First confrontation with the diagnosis

Illness related psychological problem

No social support in own environment

New medical complication

accepting the frequent medical complications, the physical restrictions caused by his condition, and his "different" looks. The remaining $11(65 \%)$ patients had learned to accept their illness, although they sometimes found it difficult to cope with new illness related complications, with their physical appearance, and with the issue of decision making on reproductive options. The most reported daily life difficulties of the patient group included the problems of clothing, of driving a car, of recognising people in the street, or watching television. The major concern of the female patients was the dual risk associated with child bearing, namely the risk of endangering their own life and the risk of transmitting the genetic defect to their progeny. One woman therefore had been sterilised. At the time of the study, three of the seven female patients were considering pregnancy but were hesitating for different reasons. One feared that her child would be more severely affected than herself and had moral objections to termination of pregnancy. A second patient had doubts because of the high genetic risk involved. The third, who had already had aortic surgery, was concerned about her own health.

\section{Compliance}

All patients reported compliance with their regular medical visits and did not experience them as a particular burden. Most patients (or their parents) relied on the hospital's invitation to be reminded of their yearly follow up. One female patient who had twice had cardiac surgery was still very anxious at each cardiological visit. All 10 patients were compliant with their medication. Five young men (two from one family) admitted that their mother had to remind them. Three (18\%) males between 17 and 18 years had difficulty accepting the physical restrictions they were subjected to. Although they were advised by their physicians to limit physical activity, they still participated in sports such as competitive hard running, basket ball, or indoor wall climbing. The remaining $14(82 \%)$ patients were compliant

Table 4 Results of the BDI, STAI, and UCL expressed as mean scores (SD) and p value for the male $(n=10)$ and female $(n=7)$ group

\begin{tabular}{lllll}
\hline & Mean male (SD) & p value* & Mean female (SD) & p value* \\
\hline $\begin{array}{l}\text { BDI } \\
\quad \text { Level of depression }\end{array}$ & $6.0(5.2)$ & & $11.3(8.2)$ & \\
STAI & $33.0(11.1)$ & NS & $40.7(16.1)$ & NS \\
$\quad$ State anxiety & $37.4(15.8)$ & NS & $42.2(6.7)$ & NS \\
$\quad$ Trait anxiety & $18.1(3.6)$ & NS & $17.3(3.6)$ & NS \\
UCL & $20.1(4.0)$ & $<0.001$ & $16.7(3.9)$ & NS \\
$\quad$ Active coping & $18.9(5.1)$ & $<0.001$ & $17.5(4.3)$ & NS \\
Palliative coping & $11.1(2.7)$ & NS & $12.3(5.3)$ & NS \\
Avoidance & $13.4(3.9)$ & $<0.01$ & $13.5(4.8)$ & NS \\
Social support seeking & $6.1(1.2)$ & NS & $5.3(2.2)$ & $<0.05$ \\
Depressive reaction & $12.6(3.1)$ & NS & $15.3(1.6)$ & $<0.05$ \\
$\quad$ Expression of emotions & & & & \\
Comforting thoughts & & &
\end{tabular}

^Student's $t$ test. but they all complained that Marfan syndrome interfered with their leisure activities.

\section{Impact of the disease on school history and career} choice

Eight (47\%) subjects reported that their school history was influenced by the illness: two attended a special school for visually disabled children, three were sent to a school for special education because their visual problems were denied and they were labelled as "intellectually impaired", and one girl followed evening courses, because day school was physically too exhausting. Two other patients reported that Marfan syndrome had played an important role in their educational choices because of the limited occupational options. All six participants who had a job experienced their visual deficiency/physical exhaustion as a great disadvantage.

Eleven patients $(65 \%)$ remembered a period in childhood during which they were teased and bullied by peers because of their tall stature, long and skinny legs, or thick glasses. Teasing varied from verbal bullying, being laughed at, to even total exclusion from the group. All felt emotionally hurt by this. Among those who had never been teased $(n=6)$, five did not have the typical marfanoid physiognomy and one girl grew up in a protected family environment.

\section{Self-image}

Nine (53\%) patients reported low self-esteem because of their physical appearance; as a result, one patient became so aggressive that he temporarily needed psychiatric treatment. Most of the patients $(n=16)$ said they were socially introvert. They had few social contacts although they liked being among other people. They blamed it on a lack of self-confidence.

\section{Professional guidance}

Thirteen $(76 \%)$ patients felt it very important to receive accurate information about Marfan syndrome. Of the remaining four patients (24\%), a 19 year old male felt he was too young to learn about all the complications he might be confronted with later on in his life; the remaining three (two from one family) were not really interested in receiving information on Marfan syndrome. All participants were unanimous on the usefulness of psychological support, available at the Genetics Department, but different reasons for using this service were given (table 3 ).

\section{PART II: PSYCHOLOGICAL TESTS (TABLE 4)}

The mean level of depression as measured by the BDI was within the minimum range for the male group (score 0-9) and within the mild range for the female group (score $>9$ ). Mean scores of the STAI showed higher levels of anxiety for state (situational) and trait (general) anxiety in the female group compared to the male group, without being significantly higher than the respective control populations. The UCL measures coping behaviour. In stressful situations, the male group showed significantly more palliative (or stress reducing) coping 
behaviour, more avoidance, and more depressive reactions than the male control population. The female participants used significantly more comforting or reassuring thoughts and showed significantly less expression of emotions compared to women in the control population. They also showed more avoidance (or denial) and more depressive reactions, but scores were not significantly different from the female control group.

\section{Discussion}

In this study, we evaluated how the life of patients with Marfan syndrome was affected by the illness. So far, few or no studies have been done on this subject, ${ }^{14}{ }^{15}$ although it can be assumed that considerable psychosocial challenges are associated with the disorder. Most patients experience significant physical limitations that interfere with daily functioning. The phenotypic appearance often leads to teasing and stigmatising, resulting in a diminished level of self-confidence. The risk of transmitting the defect to progeny represents a major concern. Therefore, it was reassuring to see that in our study most of the patients coped well with Marfan syndrome, despite particular difficulties. Successful coping behaviour results in normal psychosocial adaptation, which implies effective functioning at home, in school, and with peers. Still, it is reasonable to assume that the diagnosis of a serious disease will bring about major changes in daily life. ${ }^{16}$ Major areas of impact in our patient group were the uncertain future, disruption of body image, social and interpersonal difficulties, and limited job opportunities. The female participants were more concerned about the risks associated with child bearing than the male group. This may also explain the higher depression and anxiety levels in the female group.

In coping with emotional distress, the patient group used the typical psychological defence mechanisms observed in chronically ill young patients, namely denial and isolation. ${ }^{17}$

Compliance with medication was overall very satisfactory. It has been shown previously that the patient's previous experience with the illness, either his own or that of a family member, as well as the fact that the illness is being perceived as a serious condition, both contribute to improved compliance with medication. ${ }^{18}$ With regard to the physical restrictions, it is remarkable that the three patients being non-compliant were all 18 year old male adolescents, one of whom had a severe aortic dilatation. In the absence of any physical discomfort of a disorder, it can be difficult for patients to comprehend the reasons for restrictions imposed on them.

The fact that most patients are eager to be well informed about the disease stresses the importance of physicians' knowledge about Marfan syndrome. The unanimously positive attitude towards psychological support proved that patients need somebody to talk to about their fears, their insecurities, and their worries.

The higher level of depression and anxiety in the female group can be explained by another factor: the concept of self-image. Female adolescents may believe that their looks are strongly related to social acceptance. A study by Zeltzer et $a l^{16}$ also showed a significantly higher impact of illness upon physical appearance in female than male adolescents.

The low significance rate of the anxiety level compared to the normal population may be explained by the small sample size. Indeed, the small patient group is an important limitation to this study. This leads to the possibility that our participants represent an overestimation of well adapted young people, while the patients who refused to participate might not be coping so well with the consequences of Marfan syndrome. With regard to the psychological counselling, we think that it would be advisable that school counsellors or psychologists who are confronted with a person with Marfan syndrome should first get informed about the disease, since the psychological problem mostly will be illness induced. Education can be provided through published reports on this topic or by contacting the genetic centre or the patient support group, who will provide the mental health professional with accurate information. Psychological counselling can also be offered to young children, especially when a serious behavioural or emotional problem is observed.

It was surprising to note that almost half of our subjects were diagnosed only after the age of 12 years. This shows that patients with Marfan syndrome can still be misdiagnosed for many years or be treated for only one medical problem, while the other clinical symptoms are neglected or overlooked.

We deliberately chose to study adolescents and young adults in order to draw age specific conclusions. Further research is needed to compare these findings with data from different age groups.

Finally, connective tissue disorders related to Marfan syndrome, such as Ehlers-Danlos syndrome, may also impose serious psychosocial challenges on the patients. Since the clinical symptomatology differs from that of Marfan syndrome, it would be very interesting to explore and compare the specific problems of psychosocial adaptation in patients with this disorder.

1 De Paepe A, Devereux RB, Dietz HC, Hennekam RCM, Pyeritz RE. Revised diagnostic criteria for the Marfan syndrome. Am $千$ Med Genet 1996;62:417-26.

2 Pyeritz RE. Marfan syndrome and other disorders of fibrillin. In: Rimoin DL, Connor JM, Pyeritz RE, eds. Principles and practice of medical genetics. 3rd ed. New York: Churchill Livingstone, 1996:1027-66.

3 Pyeritz RE, Mc Kusick VA. The Marfan syndrome: diagnosis and management. $N$ Engl F Med 1979;300:772-7.

4 Dietz HC, Cutting GR, Pyeritz RE, et al. Marfan syndrome caused by a recurrent de novo missense mutation in the caused by a recurrent de novo misse
fibrillin gene. Nature 1991;352:337-9.

5 Dietz HC, Pyeritz RE. Mutations in the human gene for Dietz HC, Pyeritz RE. Mutations in the human gene for fibrillin-1 (FBN1) in the Marfan syndrom
orders. Hum Mol Genet 1995;4:1799-809.

orders. Hum Mol Genet 1995;4:1799-809.
Kainulainen K, Pulkkinen L, Savolainen A, et al. Location on chromosome 15 of the gene defect causing Marfan syndrome. N Engl f Med 1990;323:935-9.

7 Marsalese DL, Moodie DS, Vacante M, et al. Marfan's syndrome: natural history and long-term follow-up of cardiovascular involvement. $\mathcal{f} \mathrm{Am}$ Coll Cardiol 1989;14: 422-8.

8 Beck AT, Steer RA. Beck Depression Inventory manual. San Antonio, Texas: Harcourt Brace, 1993. 
9 Beck AT, Ward CH, Mendelson M, Mock J, Erbaugh J. An inventory for measuring depression. Arch Gen Psychiatry inventory for $1961 ; 4: 561-71$.

10 Spielberger CD, Gorush RL, Lushene RE. STAI manual for the State-Trait Anxiety Inventory. Palo Alto, California: Consulting Psychologists Press, 1970.

11 Van der Ploeg HM. Validatie van de Zelf-Beoordelings Vragenlijst (een nederlandstalige bewerking van de Spielberger State-Trait Anxiety Inventory). Ned Tijdschr Psychol 1980 35:243-9

12 Westbrook MT. A classification of coping behaviour based on multidimensional scaling of similarity ratings. $\mathcal{f}$ Clin Psychol 1979;35:407-10.

13 Schreurs PJG, Tellegen B, Van de Willige G. Gezondheid, stress en coping: de ontwikkeling van de Utrechtse Coping Lijst. Ned Tijdschr Psychol 1984;12:101-17.
14 Schneider MB, Davis JG, Boxer RA, Fisher M, Friedman SB. Marfan syndrome in adolescents and young adults: psychosocial functioning and knowledge. $\mathcal{f}$ Dev Behav Pediatr 1990;11:122-7.

15 Child A, Hicks J. Psychosocial aspects of Marfan syndrome. Am $\mathcal{f}$ Med Genet 1989;32:249A.

16 Zeltzer L, Kellerman J, Ellenberg L, Rigler D. Psychologic effects of illness in adolescence. II. Impact of illness in adolescents - crucial issues and coping styles. $\mathcal{F}$ Pediatr

17 Mattsson A. Long-term physical illness in childhood: a challenge to psychosocial adaptation. Pediatrics 1972;50: 801-11

18 Becker MH, Nathanson CA, Drachman RH. Mothers' health beliefs and children's clinic visits: a prospective study. $f$ Community Health 1977;3:125-35. 